\title{
ConcertTalk: A Weekend With a Portable Audio Space
}

\author{
Henry B. (Hank) Strub \\ Interval Research Corporation \\ 1801-C Page Mill Rd. \\ Palo Alto, CA 94304 U.S.A. \\ +1-415-842-6088rstrub@interval.com
}

\begin{abstract}
This paper describes a study of the ConcertTalk system, a portable audio space that was used for two days. Two-way radios were provided to attendees at Lollapalooza rock concerts, and their use was recorded and analyzed. The study's participants quickly began using the system, used it throughout the day, and found many creative ways for the radios to improve the Lollapalooza concert experience. ConcertTalk's results suggest the utility of studying CHI technologies outside of work places, both to validate and expand the foundational theory of our growing domain of knowledge in practice.
\end{abstract}

KEYWORDS Portable user interfaces, Speech \& voice, Social issues, Audio space, Observational study.

\section{INTRODUCTION}

The ConcertTalk system involved giving two-way radios to Lollapalooza concert attendees. The motivation for this study was to explore a different setting for a media space, which was specifically implemented as a portable audio space. Lollapalooza brings together national and local alternative rock in outdoor venues that hold 20,000 - 30,000 people [13]. The data for this study are recordings of ConcertTalk traffic and interviews of ConcertTalk users after the concerts. Its two sets of users liked the system and used it extensively. This study is also notable for being of an audio-only media space (an audio space).

The ConcertTalk study created an audio space with unique properties as a media space. ConcertTalk was a portable and wireless system, designed for a recreational setting without structured tasks to be performed. ConcertTalk was used intensely, although each user group had only one day with the system.

This paper begins by looking at two widely-held beliefs about media spaces that this study challenges. It then describes the study, and unique characteristics of running a study at Lollapalooza concerts. Results are presented descriptively. Special attention is devoted to creative uses of the system. The discussion suggests that foundational theory on media spaces can be expanded through broadening the domains of media space use.

\section{MEDIA SPACES: FOR WORKPLACES, WITH HIGH-} QUALITY AUDIO

Media spaces tend to support visual and audio communication, and often let users directly present, annotate and edit documents on computers. The users of media spaces are either not in the same place, not available at the same time, or both. The workplace or the school have been the assumed domains for the media space since its inception [3]: this is one belief about media spaces that is challenged in this study.

Most of the previous studies on media spaces have either been directly related to work of some sort or have simulated work situations $([3,11]$ both include reviews). Rather than look at a media space as a work tool that functioned as a "medium for social activity" ([3], p. 39), the current study found a social activity and inserted an audio space to see what behaviors would emerge. Technology envisionments (two instances were from AT\&T and Microsoft) portray videoconferencing for social situations, and low-end videoconferencing is being marketed to consumers-but these domains have not been the subject of media space studies.

As an audio space's use depends on its communication being comprehensible, one issue to investigate was whether ConcertTalk's use would be negatively influenced by its low-quality audio. Having high quality audio is considered important for media spaces, even more important than having high-quality video $[2,3,14]$ : this is the second belief about media spaces that is challenged in this study.

In the system studied by Tang and Isaacs [14], most of the audio problems were caused by delays, echo, and incomprehensible audio. Although their users were generally unwilling to wear headsets that would have reduced audio problems, the users would run the system with telephone-quality audio (which reduced audio bandwidth and decreased the overall quality but minimized delays and echo). Bly, Harrison, and Irwin [3] mention further audio problems caused by room noises like fans, and the inability to hold side conversations through a media space. 
Although most experimental media spaces have been used and studied in workplaces, they have not been used only to discuss work-related tasks. Hindus, et al. [6] had much less task-oriented interaction on the system they studied than expected, though this may have been a function of their users' work style. Hindus et al.'s system use also depended on having a critical mass of users, often influenced by the presence or absence of key participants.

These media space issues were relevant to ConcertTalk, as were issues of privacy and accessibility to other users as well as to non-users. In fact, non-users' reactions to ConcertTalk influenced its use and judgment. Also, ConcertTalk's low-quality audio actually had advantages for its use at rock concerts.

\section{THE STUDY}

The ConcertTalk study took place at Lollapalooza rock concerts, one weekend in August 1994 in Mountain View, California. Roughly ten concert attendees per day received portable two-way radios. The initial goal for the project was to see how these attendees used the radios. Its methodology was motivated by Flanagan's Critical Incident Technique [7], looking for repeating patterns and exceptions in the data.

All radio communications were recorded, except during a technical problem that lasted for less than an hour on the second day. Most of the participants were interviewed two days after the show they attended. The study's participants were reminded that their radio use was monitored and that radio transmissions recorded. Participants also knew that anyone with a scanner could listen to their communications.

\subsection{The Setting}

Lollapalooza concerts were an interesting setting for a portable audio space. There was much to see and do over the course of a concert day; shows lasted ten hours with two stages of music (sometimes both stages had bands playing concurrently). Concert venues hosted vendors representing local organizations; selling food, drink, and clothing; or offering opportunities to participate in arcade-like activities. One other activity was the Electric Carnival-a tent with art-oriented multimedia that was free to attendees [10].

Lollapalooza attendees were an interesting user community for trying ConcertTalk. A majority of them were in their late teens, which is a social time of life [5]. Teens get stereotyped as spending a lot of time talking to friends both in person and on the phone. The author expected that they might find creative uses for ConcertTalk. Also, there were questions about how ConcertTalk users and the people around them would react to the presence of radios.

\subsection{The Subjects}

Different groups of subjects used the radios each day of the study. The two groups of participants will be referred to by the day on which the people used the system: Day 1 and Day 2 participants. The goal in recruiting subjects was to find groups of teenagers in their "last year" of high school. Groups of friends were recruited through the author's coworkers. Parental release forms were obtained for participants younger than 18 years old.

The ten Day 1 participants all knew each other before the concert. All of Day 1's participants were given lawn tickets in return for their participation, and therefore none of them had access to reserved seating sections of the amphitheater. As result, Day 1 participants were expected to spend much of the day with each other. Most of the Day 1 participants had recently graduated from high school. They ranged from 17 to 20 years old; six were female and four were male.

The relationships among Day 2 participants were more complex. The main group consisted of four people who had reserved seats, along with five who were given lawn tickets. Two other teens, who were strangers to the rest of the group, were provided with lawn tickets. In the larger group, six participants were male and three were female; in the other pair one was female and one was male. Their ages ranged from 16 to 21 . Only ten radios were available for participants to use. The three young women in the main group (who spent the day together) volunteered to share two radios between them. The couple showed up late and kept the radios only part of the day.

\subsection{Audio Equipment}

Fourteen Motorola GP-300 radios were rented for this study. Figure 1 shows a ConcertTalk participant wearing a radio. The radios were of noticeably lower audio quality than telephones ${ }^{1}$. The radios, which can handle up to eight different channels, were configured with just two channels to ease analysis and to minimize the chance that users would split up on different channels and not find each other. Remote speaker/microphone units, which plugged into the radios, were given to participants. When using remotes, people with a radio could attach the radio to their waist or store it in a pack while keeping the speaker/microphone at hand or clipped at shoulder level. Two radios were used for recording the two channels, and the final two radios were used by researchers to monitor ConcertTalk traffic in case the study's participants needed assistance.

\section{OVERALL USAGE RESULTS}

The radios were well used throughout the time that participants had them. On Day 1, participants arrived at 3 p.m. Except for one twenty-minute period of silence the

'These radios have a rated audio bandwidth of $300-3000 \mathrm{~Hz}$. This $2700 \mathrm{~Hz}$ bandwidth is substantially narrower than the $3300 \mathrm{~Hz}$ bandwidth of telephone service in America. 
radios were almost constant used until they were collected at 9 p.m.. On Day 2, participants used the radios from 1 p.m. until 9 p.m., but there were a few silent periods that lasted longer than ten minutes. All cases of extended silence coincided with musical sets by popular performers-the ConcertTalk participants apparently ignored the radios only to listen to music.

New conversations commonly began less than a minute after others ended, and it was rare to have silent periods of longer than two or three minutes. Most conversations, on both days, were on one channel. There was only one period where two or three participants switched to the alternate radio channel, stating it was because they could not carry on a conversation due to too much conversation on the primary channel.

On both days, participants needed little encouragement to use the radios. The only instruction necessary was on the need to engage the radios' transmit button for perhaps half a second before the radio would start transmitting.

Although not large or heavy, the radios (along with the

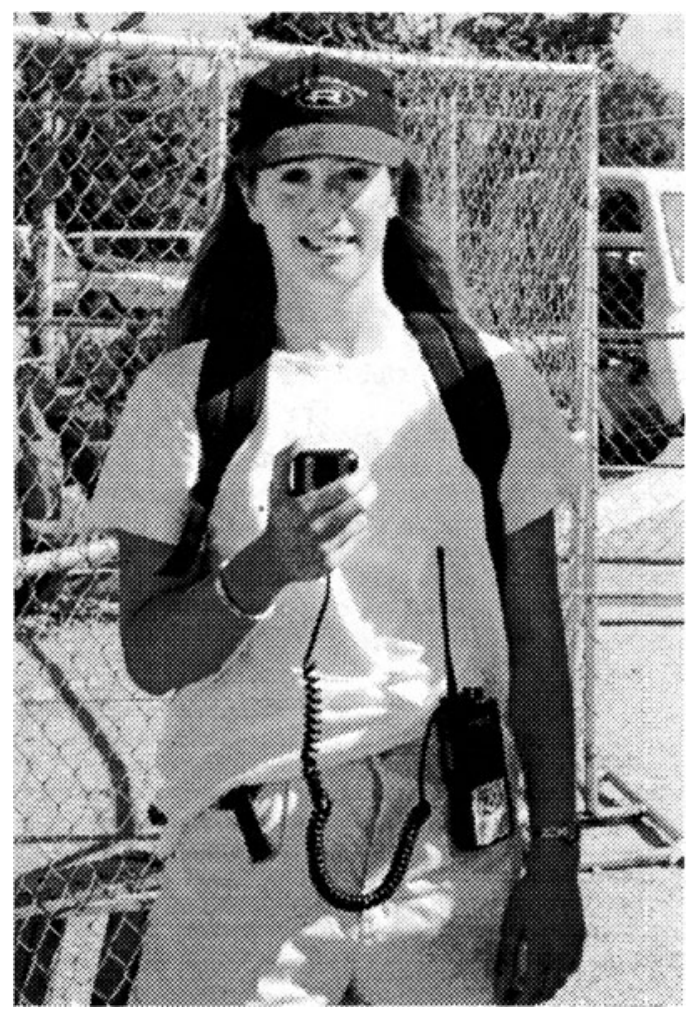

Figure 1. A ConcertTalk participant with a radio. remote microphone/speaker assemblies) looked like technology worn by police and security guards. One question was how concert attendees, both participants and the people they met, would react to the radios. There was concern that these radios might be considered "nerdy," and that participants might resist wearing and using the radios or hide the radios.

The ConcertTalk study's participants did not mind carrying radios with them. In fact, they commented on how other people thought of them as undercover security and treated them with more respect than they were used to at a rock concert. Users said it was easy to traverse through crowds, and that no one complained when someone with a radio accidentally stepped on a blanket that demarcated a group's sitting space.

\section{COMMON USES FOR THE RADIOS}

Most of the conversation on ConcertTalk was about people, mainly the participants themselves but sometimes other friends. Discussion of facts (even information exchange about the concert) was rare. As a result, most of the categories in this analysis relate to human interactions as opposed to informational categories.

\subsection{Identifying Individuals}

After learning to use the radios, initial discussions on both days were devoted to finding people and identifying when specific individuals were listening. When beginning a set of interactions, participants usually began by asking if a specific individual was listening (or "on"). Much less common were requests directed to a location, for example, a call to "anyone at home base." Participants did not respond to the few requests for everyone listening to identify themselves. It is unclear whether no one was listening at those times or whether participants did not care to identify themselves.

\subsection{Discussing Locality}

A radio conversation was usually started for some purpose (often a social one). Once a requested individual was identified as listening to ConcertTalk, participants usually determined each other's whereabouts. Knowing location often had practical value in deciding whether or where to meet. For example, some participants often used the system to find who (of the few participants of drinking age) was near a beer vendor.

A common basis for discussing locality was to coordinate getting together. On both days, participants set up blankets on the lawn so all could sit together. That was the most common place to gather, though participants also met by the "Ice Cold Beer" sign at the top of the lawn. One day's participants met in front of a landmark they called "the outhouse" (this structure was centrally located on the lawn), while the other day's participants met by a prominent yellow balloon. 
Both days' participants had to establish common frames of reference to find each other. For instance, participants had to agree whether "to the left of the stage" referred to left from the perspective of looking out from the stage or from the field looking at the stage. These discussions arose both for finding people on the field and for meeting at exits from the amphitheater.

\subsection{Arranging Activities}

Another popular use of the radios was arranging to do something together. Participants commonly did not use the radios to explain why they wanted to meet up with someone else (which in itself is interesting). When a meeting's purpose was mentioned, it was often eating. On both days, there were arrangements about possibly illicit activities. On one day, participants used code words while on the other day they openly discussed these activities.

\section{CREATIVE USES OF THE RADIOS}

Although other authors have reported on media spaces used for social interactions (especially [6]), ConcertTalk's focus was social interactions. Its social use was especially interesting for the variety of creative and fun ways the radios were used.

\subsection{Pretending to Work for Security}

All the participants in this study said they enjoyed other concert attendees perceiving them as working for security, often talking or reacting to them as undercover security or even police officers. Concert attendees spontaneously asked ConcertTalk participants whether they worked for security, or what they were doing with radios. In a few cases, participants were confronted by attendees for trying to "sneak around" the concert as undercover security. Many participants described situations where people reacted to them upon seeing the radios, before anything was said. The recordings contain cases where participants started using radio codes that sounded official, like when a participant started using codes after announcing he was going to the restroom, "521 mission found, ready to complete mission, over."

Both days' participants took advantage of the common perception of their working for security. Many described the ease of moving through crowds when radios were visible or audible. Both days' recordings have examples of participants speaking as security might, asking for "backup" or describing the location of "a bad situation" (in one case, participants approached an inebriated person who was obviously unwell). A Day 1 participant brought a flashlight to the concert, and said she used it at night with her radio to startle people as she walked through a crowd. Some Day 2 participants described how announcing "security coming through" would get people to move out of their way.

\subsection{Guest Radio Users}

On both days of Concert Talk, people not formally participating in the study tried the radios. On Day 1 , where the participants all knew each other, guests tended to be friends of a participant. Some Day 1 guests were only acquaintances of the participant whose radio they used; these guests tried to contact their friend who was on another radio. On Day 2 the participants were not as familiar with each other, and most of the guest users seemed to have just met the person whose radio they used.

Day 2's guest users were varied. For example, a young woman described a "hot looking guy" their group had met. In this case, the radios served the social function of enabling flirting. This whole transaction is otherwise incongruous since the people listening (all were male) knew the young women and probably did not care about their meeting a cute guy. Another fellow, nicknamed by participants as "the Motorola guy," used the radios to give a short monologue on his appreciation of Motorola and Motorola radios.

Two other guests during Day 2 were also noteworthy. At one time, the young women said they had met a "cute woman" (named Candy) they "wanted to introduce to the radios." During the post-concert interview, they said they had tried to set her up with a male participant. The male participants did not express interest in meeting her. The other interesting guest on Day 2 was Mark, introduced as a participant's brother. The young women wanted to meet him since he sounded "cool and together." However, Mark was an imaginary person, made up by the male participants. During the post-concert interview, Day 2's female participants stated their disappointment upon learning that Mark was not a real person.

\subsection{Development of a Group Lexicon}

Both sets of participants fell into a unique radio lexicon that seemed consistent with how radios are portrayed in the media. For example, both days' participants commonly ended transmissions with "over" to signify that they were done and waiting for a reply. "Do you copy?" was a standard way for Day 2 users to ask whether someone was listening, while Day 1 users varied between saying "copy" and "come in." When transmissions were unintelligible, it was common for Day $2 \mathrm{~s}$ to request repeat of transmissions by saying "come again."

On both days, there was an interesting evolution of language to describe where participants had set up a meeting place on the lawn. Each day began with detailed descriptions of the meeting place's location and how to find it. As the days progressed, the directions became ever shorter. This evolution of contracted descriptions, from phrases to a few words has been identified as a general phenomenon in human communication[4].

\subsection{Miscellaneous Uses}

Some of the participants' creativity was exhibited in poking fun at, and having fun with, other participants. For example, Day 2's transmissions began with the first participants annoying the person who organized their 
group while he waited outside the concert venue to distribute tickets as others arrived. Another early activity on Day 2 was spontaneous calls of "Red Leader, Red Leader." Two of Day 2's participants started the day by referring to each other as "Woodchuck" and "Gray Squirrel." It is not known whether these nicknames had been used prior to the concert.

There are also instances of spontaneous dialogues related to commercial entertainment, especially among Day 2's participants who often acted out dialogue from the movie Top Gun and the television show Star Trek. An odd phenomenon during Day 2 were infrequent requests to "maintain radio silence." Apparently, only one user uttered this statement, commonly between conversations but at least once during a dialogue.

On both days, there was radio discussion about specific participants heading off to restroom facilities. In particular, Day 2 participants repeatedly announced their entrance and when "mission completed, leaving structure," twice transmitting the flushing of toilets.

Two other unique radio events took place during Day 2. In one, participants pretended they were security asking for backup to help with a "riot" that started when a "new kid on the block" was spotted. This was followed by description of a fellow needing a "nose cast" due to "hitting his nostril with a pedal while riding a unicycle." Later in the day, it sounded as if security used ConcertTalk's channel 2 to request assistance to get a duck off the field. This was an odd event since it sounded serious and was not followed by joking. Even during the post-concert interview, one participant admitted to thinking the duck incident had been real.

\section{CONCERTTALK RADIOS AS ARTIFACTS}

So far, this paper has described how radios were used by the participants in social activities and in enjoying the concert. This section looks at how these radios' size, form, features, and characteristics worked for the ConcertTalk participants.

\subsection{Radios as Fashion}

The Lollapalooza audience is known for being fashion conscious [13]. The Motorola radios used in this study were rugged commercial devices, not at all designed for the fashion or style of Lollapalooza. Even so, neither day's participants commented about the radios looking unfashionable. Comments from non-participants about the radios were always oriented towards their purpose and why they were being carried, rather than to their appearance. The radios' practical styling did not seem to negatively influence the participants' attitudes toward their use of the radios.

\subsection{Use of the radios' multiple channels}

This section reviews observations regarding how ConcertTalk participants took advantage of the two channels they were configured with. On Day 1, participants were told that they could request assistance on channel 1. They interpreted this as a request not to use channel 1 except for emergencies, and Day 1's radio use was restricted to channel 2. Day 2's participants were told that researchers would monitor both channels, though each radio was set to channel 2 before it was distributed. There was little radio traffic on channel 1 during Day 2 other than rare instances when a participant spoke to a researcher, though on one occasion some participants wanted to carry on a dialogue when others were already talking. On that occasion, another participant interrupted them on channel 1. Perhaps that person was monitoring channel 1 , or switched channels when the others switched to channel 1 .

\subsection{Radios as instruments of safety}

Women on both days described that the radios made them feel safer at the concert. If a problem arose, they knew they could call to nearby friends for help. During both post-concert interviews, women talked about walking off by themselves, which would have made them uncomfortable had the radios not linked them to friends. On Day 2 one woman walked away from her friends in a "test loss" to get experience using the radios to find each other.

\subsection{Radios as Portable Artifacts}

The radios used in this study were too large to be carried in a pocket comfortably. Besides the radios and their antennas, ConcertTalk participants were also supplied with a remote speaker/microphone connected to the radios by means of a coiled cord.

Still, no users complained about the weight or size of the radios; in fact some users said the radios were easier to pay attention to since they had bulk. About $25 \%$ of the users reported carrying the radios in backpacks. Three or four users carried the radio in their hands and used it without the remote speaker/microphone assembly.

Many participants did comment that the remote speaker/microphone units were less comprehensible than the radios. This influenced some to not use the remote units, even though they had to hold the radios in their hands during use, and they still needed to carry the remotes all day.

Although this study demonstrates that radios can be enjoyable and easy to use, there is other evidence for this point. There are many types of radios (in the U.S. they include Citizen Band, GMRS, and other low-power FM handhelds), which are popular among many communities of users[9]. Also, Julian Orr has studied the adoption and use of radios among copier technicians [12]. The radios Orr studied were well liked and judged as contributing to more efficient repair work. 


\section{DIFFERENCES BETWEEN DAY 1 AND DAY 2}

When a study only has two different user groups, it is hard to know the basis for performance differences between them. This section discusses group differences and their implications. Day 1's participants all knew each other to some degree, and were of similar ages. Day 2's participants were more heterogeneous in age and each one in the main group knew only three or four of the other participants. Additionally, Day 2 included two other users who were strangers to all the other participants.

Day 1's conversations reflect its participants' familiarity with one another. There was a fairly constant stream of conversations, which were usually for practical purposes. Day 2 users' conversations contained more instances of fun and creativity. Perhaps this was due to their not knowing each other well. The radios may have been a medium for showing off and impressing others. A few Day 2 participants said ConcertTalk helped them get to know the other participants.

An interesting Day 2 phenomenon was the tendency for only a few people to talk at any time. The active participants divided themselves into three or four groups. In the post-concert interview, they said that all could hear incoming transmissions but only one member of any group would tend to respond.

The couple on Day 2 who were strangers to the rest of the group never became integrated into conversations; in fact, they returned their radios early. In the post-concert interview, one said it was hard to break into conversations due to not even knowing people's names.

An interesting sequence of dialogues during Day 1 was due to a unique event: the headline rock group Smashing Pumpkins played a surprise set on the second stage. A series of conversations preceded this event. It was first mentioned at 6:15 p.m. as "a surprise gig" by the group Porno for Pyros, which would start at 6:30 on the second stage. This led to excited conversation about meeting at the second stage. The first participants announced their arrival there at 6:30, but saw the equipment from another group being broken down. Discussion continued about Porno for Pyros, and some of participants met while others gave up and requested that radios be used to announce when the group showed up. About 7:00, a rock group's equipment was being set up. Word went out that the surprise group was the Smashing Pumpkins, but some of the radio traffic argued over which group was actually playing.

8.1. Consciousness about being recorded

There were early comments during Day 2 about the recordings being monitored. Later in the day, a Day 2 participant spoke to the researchers (on channel 2, which was Day 2's main channel for conversations) about not being able to see the concerts. Additionally, a Day 2 participant apologized to the person listening to the recordings for missing a great concert. Although Day 1's participants did not talk about being recorded or monitored, their early use of code words for illicit activities may have reflected planning regarding ConcertTalk's use being recorded.

\section{CONCERTTALK'S AUDIO QUALITY WAS
ACCEPTABLE}

The radio's poor audio quality did not appear to impair ConcertTalk's use. Retransmission requests occurred only when transmissions were made with loud background noise, or when statements were not transmitted due to participants starting to speak immediately after engaging the radio transmit button. In the post-concert interviews, no one complained about the radios' audio quality. Participants said they had no problems with comprehensibility. On both days, participants seemed to have no difficulty identifying each other.

\subsection{Being Loud Enough at the Right Time}

Ideally, a portable communication system should always be at the right volume for whatever its users are doing. This was not the case with ConcertTalk. Some participants turned off their radios when they listened to music, making them unavailable to other participants. Others turned the radio volume up when they were in a noisy situation, but then were embarrassed by the radios being loud (and calling attention to themselves) just after they left the noisy area. Yet, while sitting on a blanket in the concert area, a few participants found it easier to use the radios to communicate with someone sitting four people away rather than talking directly to those people.

\subsection{Mediocre Audio can have Benefits}

The radios' low audio bandwidth might be considered a feature for the Lollapalooza setting. In the midst of the noisy ambient background of a festival rock concert, radio transmissions had a distinct sound. As long as radios were loud enough to be audible over the ambient noise (that was not always the case, especially when participants were close to an active stage), transmissions were distinctive. Had radios provided better quality, participants may have had trouble distinguishing transmissions from the live acoustic environment. Although this phenomenon should not be taken as a recommendation for poor audio, there should be consideration of making media space communications distinctive from those in their users' local spaces.

\section{CONCERTTALK AND MEDIA SPACES}

This paper began by raising questions on whether siting an audio space outside of a workplace might inform the theoretical foundation of media spaces. This final section discusses ConcertTalk as a media space.

Using ConcertTalk, someone speaking had no feedback from listeners except after ending a transmission, and after a listener responded with a transmission. This feature was in direct opposition to the property of seamlessness 
that is often considered a virtue for media spaces [8]. There were loud background noises around the concert site and they would have been distracting had they been constantly transmitted over ConcertTalk. The tradeoffs involved in such issues should be considered in the design of personal systems for communication.

This "push to talk" feature also helps ConcertTalk results argue for modifications to the general rule that audio quality is important in media spaces $[2,3,14]$. Participants did not complain about the system's audio quality, though they occasionally asked that transmissions be repeated. However, the narrow bandwidth and low fidelity of the radios' speakers helped participants differentiate between sounds in the world and the system's sounds. There are disadvantages to halfduplex systems like ConcertTalk (for example, it required turn taking, and did not allow interruptions of long statements). ConcertTalk was immune from the common media space problems of echo and delay.

What factors will contribute to successful use of a grouporiented audio space like ConcertTalk? The number of users can make or break a system. It is hard to have more than one conversation running on a single audio channel for more than a brief period since additional transmissions often result in the channel's becoming inaudible. A group needs to be large enough so there are responses to requests or questions, but not so large that users cannot break in to say something. Multiple radio channels can be available when a primary channel is overused, but this advantage is a risk for the system: once people divide up across other channels it can be difficult to find specific people. Also, a successful system like ConcertTalk needs social users who talk but not all the time. Ten participants worked well for ConcertTalk, but it is worth investigating how the size of an audio space's user community influences its use. Hindus, et al. [6] suggest that an audio space needs a critical mass of users to be successful. The system they described needed the presence of key users to run well, though ConcertTalk had no key users.

Another issue relevant to media spaces is the possible conflict between group availability and person-specific accessibility [1]. People sometimes want to be available to a specific sub-group and still be accessible to individuals who are not part of that group. ConcertTalk's constraints and simplicity helped turn those issues inside out. Group exclusivity was established by the radios being set to reserved frequencies, and by their only going to known individuals (except for the two strangers on Day 2, who did not successfully integrate with the other users). Individual accessibility was easy to control through turning a radio's volume down, shutting it off, or simply by ignoring it. It was also easy to expand accessibility by letting guests listen and speak into a radio.
Indeed, any technological system exists within social practices that help the technology be more or less effective. Features that are advantageous in some situations will prove problematic in others. ConcertTalk was considered a success by its participants, but this may reflect properties that are tied to Lollapalooza which might not transfer to other situations.

\subsection{ConcertTalk's Intentionality and Privacy}

ConcertTalk can be said to have offered no privacy to participants, since all radio traffic was recorded and anyone with a scanner may have listened in on conversations. However, a significant privacy-related difference between the ConcertTalk radios and many media spaces is that nothing was transmitted over ConcertTalk without someone pushing a button to transmit. Although this step can be viewed as an impediment to communication, intentionality was behind every utterance that was broadcast. The intentionality of ConcertTalk utterances is in sharp contrast to media spaces that have open audio channels, which often result in users forgetting that fellow users can hear comments addressed to someone nearby (or to something, like a phone)[6]. Additionally, although participants were good at recognizing each others' voices, there was some anonymity on the system ${ }^{2}$. Finally, participants could see anyone standing nearby who might overhear ConcertTalk transmissions.

\section{METHODOLOGICAL ISSUES}

It would have been interesting to have microphones and recorders, separate from the radios, with each participant. There are records on how people coordinated activities and get-togethers, but there is no data on what happened once people met up. Also, people experience varied personal audio environments, yet there is no data on how non-radio noises interacted with radio transmission and reception. Additionally, it would have been interesting to observe participants as they decided to use radios, either were or were not successful over the airwaves, and how participants reacted to radio transmissions.

\section{CONCLUSIONS}

This study began as an exploration of an audio space, used away from work places or schools. It was expected that such a different environment would result in new questions and issues about audio spaces in particular, and media spaces in general. This study also shows the general value of studying concepts from unique perspectives.

The ConcertTalk system can be considered successful. Its participants used the system heavily and creatively. ConcertTalk contributed positively to how participants experienced Lollapalooza concerts. Much of its activity was purposeful, and can be viewed as being like work even though discussion topics were social.

\footnotetext{
${ }^{2}$ This was especially in evidence by "Mark," the imaginary brother and visitor to the system, who fooled Day 2 's females.
} 
Allan MacLean has suggested that one framework to use when analyzing a CSCW system is to see how it changes activities that people perform (personal communication). ConcertTalk was a success from this perspective in that it appears to have helped participants perform activities they would probably have already engaged in. For example, the primary ConcertTalk activity was making arrangements for getting together. Many of the other activities can be viewed as cases of flirting or showing off. The popular "playing security guard" game might appear to violate this assertion, but it is consistent from the perspective that playing security helped participants move through concert crowds, and thereby became a means for helping the participants show off.

ConcertTalk's results should not be overgeneralized. A Lollapalooza concert is a unique type of event. The day is too long for attendees to stay in one place, yet it provided a set of activities that a group could enjoy together. Common interests in similar types of information ${ }^{3}$ encouraged communication and may have in fact made the system's "party-line" nature especially useful. However, participants were not engaged in attention-demanding tasks so there were no activities to be interfered with due to radio interruptions. Even in non-work environments, it is likely that different activities will be more and less amenable to their users' being interrupted by small talk from an audio space.

The ConcertTalk study was a theoretically-based system that situated an audio space in the outside world. It was used and appreciated by its participants; in fact both sets of participants asked about the cost of renting the equipment. ConcertTalk demonstrates that some of the theory growing around media spaces is a function of their being studied only in work places, and broadens the range of issues to consider regarding audio quality in media spaces.

\section{ACKNOWLEDGMENTS}

Andrew Singer provided the spark for this project. Anthony Wagner and Lisa Hagström helped set up and run the study. Debby Hindus helped improve this manuscript. David Gessel, Bud Lassiter, Jason Lewis, and Wayne Burdick helped with technical details. Laura Dimario, Andrew Singer, Elin Pedersen, and Sue Faulkner provided contacts to participants. Noel Hirst; and staff from Shoreline Amphitheater and Lollapalooza all helped this study take place.

14. BIBLIOGRAPHY

1. Bellotti, V., S. B. Shum, A. MacLean and N.

Hammond. Multidisciplinary modelling in $\mathrm{HCI}$ design

...In theory and in practice. Proceedings of $\mathrm{CHI}$ '95:

Conference on Human Factors in Computing Systems, 1995: 146-153.

${ }^{3}$ For example, who to meet or eat with, or whether a group was still on stage.
2. Bielski, L. Bellcore's videoconferencing research: Bigger displays are less visible and more powerful. Advanced Imaging. 8: 32-34, 1994.

3. Bly, S. A., S. R. Harrison and S. Irwin. Media Spaces: Bringing people together in a video, audio, and computing environment. Communications of the ACM, 1993, 36(1): 28-46.

4. Clark, H. H. and D. Wilkes-Gibbs. Referring as a collaborative process. Cognition, 1986, 22: 1-39.

5. Cole, M. and S. R. Cole. The development of children. Scientific American Books, New York, 1993.

6. Hindus, D., M. S. Ackerman, S. Mainwaring and B. Starr. Thunderwire: A field Study of an Audio-Only Media Space. Proceedings of Computer Supported Cooperative Work '96, 1996: 238-247.

7. Flanagan, J. C. The critical incident technique. Psychological Bulletin, 1954, 51(4): 327-358.

8. Ishii, H., M. Kobayashi and J. Grudin. Integration of interpersonal space and shared workspace: Clearboard design and experiments. ACM Transactions on Information Systems, 1993, 11(4): 349-375.

9. Kneitel, T. Two-way radio: Uncensored. Popular Communications. 13: 8-9, 1995.

10. Markoff, J. An impresario's high-tech big top. New York Times. C1-C2, 1994.

11. Olson, J. S., G. M. Olson and D. K. Meader. What mix of video and audio is useful for small groups doing remote real-time design work? Proceedings of CHI '95: Human Factors in Computing Systems, 1995: 362-368.

12. Orr, J. E. Ethnography and organizational learning: In pursuit of learning at work. Xerox PARC, Systems \& Practices Lab, SPL-93-040, P93-00090, 1993.

13. Pond, S. The trick is to be loved but not embraced. New York Times. 1994.

14. Tang, J. C. and E. A. Isaacs. Why do users like video? Studies of multimedia-supported collaboration. CSCW, 1993, 1: 163-196. 